\title{
Health Coaching to Enhance Psychological Well-being Among Veterans with Suicidal Ideation: a Pilot Study
}

\author{
Lauren M. Denneson, $P h D^{1,2}$, Amira Y. Trevino, $B S^{1,2}$, Emily A. Kenyon, $B A^{7}$, Sarah S. Ono, $P h D^{1,3}$, \\ Paul N. Pfeiffer, $M D^{4,5}$, and Steven K. Dobscha, $M D^{1,2}$
}

\begin{abstract}
${ }^{1}$ Center to Improve Veteran Involvement in Care (CIVIC), VA Portland Health Care System, Portland, OR, USA; ${ }^{2}$ Department of Psychiatry, Oregon Health \& Science University, Portland, OR, USA; ${ }^{3}$ Department of Family Medicine, Oregon Health \& Science University, Portland, OR, USA;

${ }^{4}$ Department of Psychiatry, University of Michigan, Ann Arbor, MI, USA; ${ }^{5}$ VA Center for Clinical Management Research, VA Ann Arbor, Ann Arbor, MI, USA.
\end{abstract}

KEY WORDS: suicide; self-care; primary health care; patient-centered care; veterans.

J Gen Intern Med 34(2):192-4

DOI: $10.1007 / \mathrm{s} 11606-018-4677-2$

(C) Society ofGeneral Internal Medicine (This is a U.S. government work and not under copyright protection in the U.S.; foreign copyright protection may apply) 2018

\section{INTRODUCTION}

According to Veterans Affairs (VA) surveillance data, veterans are approximately twice as likely to die by suicide than civilians, and the importance of developing effective suicide prevention strategies for primary care settings is well known. ${ }^{1}$ Psychological well-being (comprising purpose in life, autonomy, personal growth, positive relations with others, environmental mastery, and self-acceptance $)^{2}$ can buffer against a range of adverse experiences. Some research has shown that psychological well-being protects against suicidal ideation and behavior among people with mental health symptoms. ${ }^{3}$ No evidencebased interventions exist that specifically aim to increase psychological well-being among veterans at risk for suicide.

Health coaching is a patient-centered modality that focuses on personal goals, values, and strengths to facilitate well-being. ${ }^{4}$ Health coaching has not been studied among those at increased risk for suicide. In this single-arm, mixed-methods pilot study, we sought to determine the feasibility and acceptability of a telephone-delivered health coaching intervention among veterans with suicidal ideation. We also examined changes in psychological well-being and other health outcomes (suicidal ideation severity, depression, post-traumatic stress, perceived stress, and anxiety) from baseline to post-intervention.

\section{METHODS}

Study methods were approved by the VA Portland Health Care System institutional review board. We enrolled 22 Post-9/11 veterans with at least one prior-year primary care visit and recent suicidal ideation (prior 3 months) into the Whole Health Coaching for Suicidal Ideation (WHC-SI) program. WHC-SI is based on the Veterans Health Administration (VHA)'s

Published online September 24, 2018 model of Whole Health ${ }^{5}$ and uses reflective listening, motivational interviewing, assessment, and accountability strategies to facilitate goal-setting, articulation of action steps, and goal achievement. ${ }^{4}$ Participants completed up to eight weekly health coaching sessions (30-60 min each) by telephone with an experienced coach (non-licensed provider).

We extracted prior-year utilization and diagnostic data from VHA patient data files. Participants completed assessments at baseline and at 4, 8, and 16 weeks post-baseline. Assessments contained Ryff's Psychological Well-Being scales to assess psychological well-being and several other health outcome measures. Using paired-samples $t$ tests, we examined changes in means from baseline to each of the follow-up time points for each of the measures. Post-intervention, participants completed the Client Satisfaction Questionnaire 8 (CSQ8) to assess perceived satisfaction with the WHC-SI intervention and participated in an hourlong, semi-structured interview with a study staff member about their experiences with the intervention. Intervention fidelity was monitored using a coaching skills checklist.

\section{RESULTS}

Of the 42 veterans reached by phone, 20 declined participation and 22 were enrolled into the study (52\% enrollment rate). Eighty-six percent $(n=19)$ of participants met intervention completion criteria ( $\geq 5$ sessions). Table 1 describes the study sample. Fidelity scores ranged from 81 to $100 \%$ (with $\geq 70 \%$ considered "maintaining fidelity"). Overall mean satisfaction with the intervention was 30 (possible score of 32). Table 2 presents mean differences in health outcome measures between baseline and each follow-up assessment. At the post-intervention assessment (week 8), we observed significant increases in psychological wellbeing $(t[12]=2.89, p=0.01)$, and significant improvements in other mental health measures.

Qualitative findings (Table 2) further supported the acceptability of WHC-SI among veterans with suicidal ideation and its potential impact on health outcomes. In summary, participants reported positive program experiences and described increases in aspects of psychological well-being. Participants' 
Table 1 Participant Demographic and Clinical Characteristics, $n=22$

\begin{tabular}{|c|c|c|}
\hline Variable & $n(\%)$ & \\
\hline \multicolumn{3}{|l|}{ Demographic characteristics } \\
\hline Age, mean (SD) & $35.5(9.5)$ & \\
\hline Male (vs. female) & $18(81.8)$ & \\
\hline Minority status (vs. white, non-Hispanic) & $7(31.8)$ & \\
\hline \multicolumn{3}{|l|}{ Marital status } \\
\hline Married & $7(31.8)$ & \\
\hline Single, never married & $6(27.3)$ & \\
\hline Divorced, separated, or widowed & $9(40.9)$ & \\
\hline \multicolumn{3}{|l|}{ Education } \\
\hline High school or GED & $3(13.6)$ & \\
\hline Some college & $12(54.5)$ & \\
\hline 4 -year college degree & $4(18.2)$ & \\
\hline More than 4-year college degree & $3(13.6)$ & \\
\hline \multicolumn{3}{|l|}{ Employment status } \\
\hline Disabled/retired & $6(27.3)$ & \\
\hline Unemployed & $6(27.3)$ & \\
\hline Employed & $10(45.5)$ & \\
\hline \multicolumn{3}{|c|}{ Prior-year mental health utilization and diagnoses } \\
\hline Any mental health care & $21(95.5)$ & \\
\hline Anxiety & $11(50.0)$ & \\
\hline Depression & $18(81.8)$ & \\
\hline Post-traumatic stress disorder & $14(63.6)$ & \\
\hline Substance use disorder & $9(40.9)$ & \\
\hline Sleep disorder & $10(45.5)$ & \\
\hline Baseline health outcome measures & Mean (SD) & Min, $\max$ \\
\hline Psychological well-being* & $3.5(0.8)$ & $1.9,4.6$ \\
\hline Suicidal ideation severity ${ }^{\dagger}$ & $3.4(2.6)$ & 0,8 \\
\hline Depression symptom severity & $15.5(5.6)$ & 5,25 \\
\hline Generalized anxiety symptoms $\S$ & $12.2(5.6)$ & 2,21 \\
\hline Perceived stress $\|$ & $32.5(9.1)$ & 17,55 \\
\hline Post-traumatic stress symptoms ${ }^{\mathrm{II}}$ & $47.6(20.0)$ & 11,83 \\
\hline
\end{tabular}

*Ryff's Psychological Well-Being scales; ${ }^{\prime}$ Suicidality subscale of the Depressive Symptom Inventory (DSI-SS); Fatient Health Questionnaire-9 (PHQ-9); ' Generalized Anxiety Disorder-7 (GAD-7); "Cohen's Perceived Stress Scale (PSS); "PTSD checklist-5 (PCL-5)

and health coach feedback suggest intervention modifications for this population: accommodating discussion of deeper topics, use of smaller goal-oriented action steps, providing additional information about available VA resources, and offering additional coaching sessions.

\section{DISCUSSION}

Few studies have examined the use of health coaching among clients with mental health conditions ${ }^{6}$ and no previously published work has examined the use of coaching among patients with suicidal ideation. Our findings supported the feasibility and acceptability of WHC-SI among veterans with suicidal ideation and suggested that WHC-SI may enhance psychological well-being in this population.

This is a pilot study without a control group; more research is needed to understand the effectiveness of WHC-SI. Generalizability of findings to other populations is limited due to our sample's size and lack of demographic diversity. However, our sample is demographically representative of post-9/11 veterans. While most participants completed the intervention, only $60 \%$ of participants completed the post-intervention assessment. Despite these limitations, this study provides evidence that WHC-SI could be a promising solutions-focused,
Table 2 Outcomes

\begin{tabular}{|c|c|c|c|c|c|}
\hline \multicolumn{6}{|c|}{ Mean differences in quantitative outcomes from baseline } \\
\hline $\begin{array}{l}\text { Outcome and } \\
\text { time }\end{array}$ & $n$ & $M(95 \% \mathrm{CI})$ & SD & $p$ & $d$ \\
\hline \multicolumn{6}{|c|}{ Psychological well-being } \\
\hline 4 weeks & $16^{\circ}$ & $0.11(0.08,0.28)$ & 0.32 & 0.18 & \\
\hline 8 weeks & 13 & $0.33(0.08,0.59)$ & 0.42 & 0.01 & 0 \\
\hline 16 weeks & 13 & $0.41(0.03,0.78)$ & 0.62 & 0.04 & \\
\hline \multicolumn{6}{|c|}{ Depression symptoms } \\
\hline 4 weeks & 17 & $\begin{array}{l}-2.53(-4.68 \\
-0.38)\end{array}$ & 4.19 & 0.02 & \\
\hline 8 weeks & 13 & $\begin{array}{l}-3.23(-6.05 \\
-0.42)\end{array}$ & 4.66 & 0.03 & 0.6 \\
\hline 16 weeks & 13 & $\begin{array}{l}-4.08(-7.82, \\
-0.33)\end{array}$ & 6.20 & 0.04 & 0.6 \\
\hline \multicolumn{6}{|l|}{ Perceived stress } \\
\hline 4 weeks & 17 & $\begin{array}{l}-2.71(-6.06 \\
0.65)\end{array}$ & 6.53 & 0.11 & 0.4 \\
\hline 8 weeks & 13 & $\begin{array}{l}-7.08(-11.80, \\
-2.35)\end{array}$ & 7.81 & 0.01 & 0.9 \\
\hline 16 weeks & 13 & $\begin{array}{l}-6.69(-13.13, \\
-0.26)\end{array}$ & 10.65 & 0.04 & 0. \\
\hline \multicolumn{6}{|l|}{ Anxiety symptoms } \\
\hline 4 weeks & 17 & $\begin{array}{l}-1.53(-3.55 \\
0.50)\end{array}$ & 3.94 & 0.13 & 0.3 \\
\hline 8 weeks & 13 & $\begin{array}{l}-3.15(-6.07 \\
-0.24)\end{array}$ & 4.82 & 0.04 & 0.6 \\
\hline 16 weeks & 13 & $\begin{array}{l}-3.62(-6.36 \\
-0.87)\end{array}$ & 4.54 & 0.01 & 0.8 \\
\hline \multicolumn{6}{|l|}{ PTSD symptoms } \\
\hline 4 weeks & 17 & $\begin{array}{l}-5.53(-11.95 \\
0.89)\end{array}$ & 12.49 & 0.09 & \\
\hline 8 weeks & 13 & $\begin{array}{l}-14.23(-22.29 \\
-6.17)\end{array}$ & 13.34 & 0.00 & 1.0 \\
\hline 16 weeks & 13 & $\begin{array}{l}-11.08(-20.78 \\
-1.38)\end{array}$ & 16.05 & 0.03 & 0.6 \\
\hline \multicolumn{6}{|c|}{ Suicidal ideation severity } \\
\hline 4 weeks & $16^{\circ}$ & $\begin{array}{l}-0.69(-1.29 \\
-0.81)\end{array}$ & 1.14 & 0.03 & 0 \\
\hline 8 weeks & 13 & $\begin{array}{l}-0.69(-1.72 \\
0.34)\end{array}$ & 1.70 & 0.17 & 0. \\
\hline 16 weeks & 13 & $-1.31(-2.88$ & 2.59 & 0.09 & 0.5 \\
\hline
\end{tabular}

Selected qualitative quotes

"I am in a lot better place. I am not drinking and using drugs, I am not sitting on the couch. I am back to who I was before...I think [this program is] going to change veterans lives and I hope I can look back ten years from now and go 'That program saved my life,' and it really did, so thank you guys."

"It's liberating. It's something that's self-improvement. It's hopeful, not hopeless."

"You are getting to know who people are as individuals; what makes them tick, what makes them what they are. You are not doing this in a psychiatric way and that's a beautiful thing. It's adding encouragement, it's exhorting one another and boosting each other up. It's being a positive sounding board to encourage people that they have a purpose, that they have drive. I think you guys are doing that to an awesome level."

"I got a direction I am going in now, and I got a path to look forward to now. Whereas before, I did not see that. I guess you could say it illuminated the path."

"I do not think the program naturally should be 8 weeks, I think it should be longer... Even if it's 8 weeks at first to really bang something out and then have a couple months where a call every month or a checkup every month or 6 months after."

patient-centered approach to support suicide prevention efforts among veterans with suicidal ideation.

ACKNOWLEDGMENTS: This material is based upon work supported by the Department of Veterans Affairs, Veterans Health Administration, and the American Foundation for Suicide Prevention project PRG0-027-15. 
Corresponding Author: Lauren M. Denneson, PhD; Center to Improve Veteran Involvement in Care (CIVIC)VA Portland Health Care System, 3710 SW US Veterans Hospital Rd (R\&D 66), Portland, OR 97239, USA (e-mail: Lauren.Denneson@va.gov).

\section{COMPLIANCE WITH ETHICAL STANDARDS:}

Study methods were approved by the VA Portland Health Care System institutional review board.

Disclaimer: Preliminary findings from this manuscript were presented at the International Academy of Suicide Research/American Foundation for Suicide Prevention's International Summit on Suicide Research in November 2017. The views expressed in this article are those of the authors and do not necessarily reflect the position or policy of the Department of Veterans Affairs or US government.

Conflict of Interest: The authors declare that they do not have a conflict of interest.

\section{REFERENCES}

1. Mann JJ, Apter A, Bertolote J, et al. Suicide prevention strategies: a systematic review. JAMA. 2005;294:2064-74.

2. Ryff CD, Keyes CLM. The Structure of Psychological Well-being Revisited. J Pers Soc Psychol. 1995;69:719-727.

3. Panagioti M, Gooding PA, Taylor PJ, Tarrier N. Perceived social support buffers the impact of PTSD symptoms on suicidal behavior: implications into suicide resilience research. Compr Psychiatry. 2014;55:104-112.

4. Wolever RQ, Jordan M, Lawson $\mathbf{K}$, Moore M. Advancing a new evidencebased professional in health care: job task analysis for health and wellness coaches. BMC Health Serv Res. 2016;16:205-016-1465-8.

5. Krejci LP, Carter K, Gaudet T. Whole health: the vision and implementation of personalized, proactive, patient-driven health care for veterans. Med Care. 2014;52:S5-8.

6. Corrie S. SGCP Research Network: What role do coaching practitioners have in working with mental health issues? Results of a survey. The Coaching Psychologist. 2017;13:41-48. 